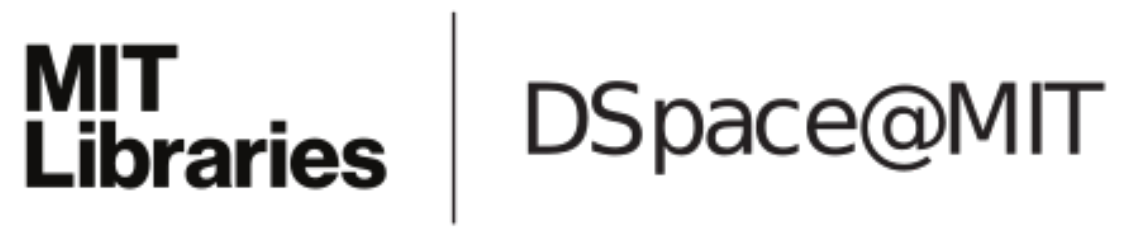

MIT Open Access Articles

Personality, Attitudes, and Bonding in Conversations

The MIT Faculty has made this article openly available. Please share how this access benefits you. Your story matters.

Citation: Jaques, Natasha, et al. "Personality, Attitudes, and Bonding in Conversations." International Conference on Intelligent Virtual Agents, IVA 2016, 20-23 September, 2016, Los Angeles, California, edited by David Traum et al., Springer International Publishing, 2016.

As Published: http://dx.doi.org/10.1007/978-3-319-47665-0_37

Publisher: Springer Nature America, Inc

Persistent URL: https://hdl.handle.net/1721.1/125247

Version: Author's final manuscript: final author's manuscript post peer review, without publisher's formatting or copy editing

Terms of use: Creative Commons Attribution-Noncommercial-Share Alike 


\title{
Personality, Attitudes, and Bonding in Conversations
}

\author{
Natasha Jaques ${ }^{1}$, Yoo Lim Kim² ${ }^{2}$ and Rosalind Picard ${ }^{1}$ \\ 1 MIT Media Lab, Cambridge MA 02139, USA, \\ $\{$ jaquesn, picard $\}$ @media.mit.edu, \\ http://http://affect.media.mit.edu/ \\ 2 Wellesley College, Wellesley MA 02481, USA \\ ykim9@wellesley.edu
}

\begin{abstract}
This paper investigates how the personality and attitudes of intelligent agents could be designed to most effectively promote bonding. Observational data are collected from a series of conversations, and a measure of bonding is adapted and verified. The effects of personality and dispositional attitudes on bonding are analyzed, and we find that attentiveness and excitement are more effective at promoting bonding than traits like attractiveness and humour.
\end{abstract}

Keywords: Personality, attitudes, bonding, rapport

\section{Introduction and Related Work}

Many studies have probed how to make intelligent virtual agents (IVAs) more appealing to human users, by focusing on the aesthetic appeal of the characters (e.g. [1]), their facial expressions (e.g. [2]), mirroring (e.g. [3]), and the contingency of their non-verbal responses [4]. Detailed models of bonding and rapport [5], and interpersonal emotions in conversations [6], have also been developed.

We contribute to this work by examining which dispositional attitudes and personality traits are most important to bonding and rapport. For example, if Agreeableness is important to rapport (as reported in [7]), it may suggest that designing the responses of an IVA to appear more kind, polite, and nonconfrontational would be beneficial [8]. The effect of personality on bonding is compared to that of traits like attractiveness and humour, to suggest which characteristics deserve the most attention when designing an IVA. We use a bonding measure adapted from the Working Alliance Inventory [9], and find that bonding is strongly related to participants' perceptions of conversation quality and interpersonal connection.

Personality has been examined in the context of users' reactions to an IVA [10], and in terms of how personality similarity affects conversation quality [7]. In some cases similarity is helpful, as when partners have a similar level of extraversion. However, interactions between two disagreeable participants were rated as the least pleasant. While this study provides valuable insights, participants were all college students, and it is uncertain how far these claims can generalize. Our study builds upon this previous work by collecting data from participants from 
a diverse range of ages, ethnicities, and backgrounds, and relating personality and conversation quality to a robust measure of bonding.

\section{User Study}

Data were collected from a study in which participants conversed while being recorded with cameras, microphones, and Microsoft Kinects ${ }^{3}$. To conceal the true nature of the study and ensure participants could act naturally, participants were told the purpose of the study was to train computer algorithms to read lips. They were asked not to over-emphasize their lip movements, and to keep the conversation flowing as naturally as possible. The interaction lasted for about 20 minutes, after which participants were debriefed. All procedures were approved by the university IRB. 30 participants (13 male, 17 female), were recruited through the MIT Behavioral Research Lab (BRL) from the wider Boston community. There was variety across participants in age $(\mathrm{M}=40.0, \mathrm{SD}=15.3)$, occupation, ethnicity, and socioeconomic status.

Participants completed both a pre- and post-study survey. Personality traits were collected during the pre-study survey using the Big-Five Factor Markers questionnaire [11]. The post-study survey contained a Perception of Interaction questionnaire similar to that of [7], asking participants to rate their partner on a Likert scale on qualities like interesting, funny, and attractive. Bonding was measured with a modified version of the Bonding subscale of the Working Alliance Inventory (B-WAI). The WAI was developed to measure the degree of collaboration and trust between a therapist and client; the bonding subscale measures positive personal attachment, including "mutual trust, acceptance, and confidence" (p. 224) [9]. Items include, "My therapist and I understood each other", and "I felt uncomfortable with my therapist". The scale was adapted to our study by substituting the phrase "my partner" for "my therapist", and removing items 17,21 , and 36 , which were irrelevant for short conversations between strangers. Two other items were modified slightly; Item 29 was changed to read "I had the feeling that if I said or did the wrong things, my partner would stop talking with me" (rather than "working with me"), and in Item 28 the phrase "my relationship" was replaced with "getting along", such that the item reads, "Getting along with my partner was important to me".

\section{$3 \quad$ Results}

\subsection{Reliability of the bonding scale}

The following analysis relies on B-WAI as an aggregate measure of the rapport and trust participants felt toward their conversational partner, as well as their feelings of warmth, comfort, and enjoyment. To demonstrate that B-WAI measures these characteristics, correlations between it and eight self-reported

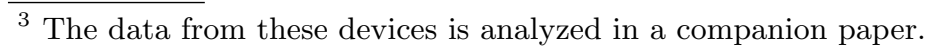


Likert-scale ratings of conversation quality were computed (see Table 1). B-WAI is positively related to participants' ratings of their partner as interesting, charming, friendly, and funny, and inversely to distant and annoying. To control for alpha inflation, a Bonferroni correction was applied; the relationships between B-WAI and interesting, annoying, and distant were still significant. Given the small sample size $(N=30)$ and relatively low statistical power, such results suggest B-WAI is strongly related to participants' perceived conversation quality.

Table 1: Pearson's $r$ correlations between B-WAI and conversation quality. Bolded measures are significant after performing a Bonferroni correction.

\begin{tabular}{lcc}
\hline Measure & $r$ & $p$ \\
\hline Interesting & $\mathbf{. 6 9 1 2}$ & $<.001$ \\
Charming & .4342 & .021 \\
Friendly & .3806 & .038 \\
Funny & .3736 & .046 \\
Engaging & .1104 & .561 \\
\hline
\end{tabular}

(a) Positive correlations

\begin{tabular}{lcc}
\hline Measure & $r$ & $p$ \\
\hline Distant & $\mathbf{- . 6 2 0 7}$ & $<.001$ \\
Annoying & $\mathbf{- . 5 5 4 9}$ & $\mathbf{. 0 0 1}$ \\
Awkward & -.2589 & .167 \\
\hline
\end{tabular}

(b) Negative correlations

\subsection{Designing an agent to promote bonding}

A multiple regression analysis is employed to determine if it is possible to accurately estimate participants' B-WAI scores from information about their partner's personality and attitudes, and to analyze how these traits affected bonding. Although we could include factors about the participant themselves in the model, this is not under control of the designer of a virtual agent. Rather, we restrict focus to characteristics about the IVA that could be modified. Only the following traits were included: partner's Big Five extraversion and agreeableness scores, extraversion match (a binary variable indicating whether the pair were both introverts or both extroverts), gender match (defined similarly), age difference, and the participant's rating of their partner on the following qualities: attractive, funny, attentive, and excited. The resulting model statistically significantly predicted WAI score, $F(9,19)=4.656, p=.004$, and was able to account for $72.4 \%$ of the variance in WAI score, $R=.851$.

Table 2 shows the coefficients of the regression model. The first column (unstandardized $\beta$ ) gives the increase (or decrease) that can be expected in bonding for a 1-unit increase in the variable. For example, an increase in a participant's rating of their partner as attentive is associated with an increase of 6.024 in expected B-WAI. Three significant effects were detected; whether the gender of the two participants matched, and whether the partner was perceived as excited and attentive. It appears that bonding will be highest when the partner's gender is not a match, the partner gives the impression of listening carefully to the participant, and the partner is enthusiastic about the conversation.

\section{Discussion and Conclusions}

We have compared the effects of personality, attractiveness, humour, and attitudes like excitement on bonding and rapport. We have found that bonding can 
Table 2: Linear regression coefficients for each of the factors in the model.

\begin{tabular}{|l|l|l|l|l|l|}
\hline Variable & Unstandardized $\beta$ & Standard Err. & Standardized $\beta$ & $t$ & $p$ \\
\hline Extraversion & -4.461 & 3.984 & -.179 & -1.120 & .279 \\
Agreeableness & -5.441 & 6.393 & -.127 & -.851 & .407 \\
Extraversion match & 3.158 & 2.169 & .235 & 1.456 & .165 \\
Gender match & -6.765 & 2.923 & -.393 & -2.314 & .034 \\
Age difference & .150 & .091 & .255 & 1.646 & .119 \\
Attractive & .352 & .788 & .067 & .446 & .662 \\
Funny & -1.624 & 1.314 & -.207 & -1.237 & .234 \\
Attentive & 6.024 & 1.251 & .847 & 4.814 & .000 \\
Excited & 1.622 & .754 & .342 & 2.152 & .047 \\
\hline
\end{tabular}

be predicted effectively using personality and the traits described. Future work is needed to determine the extent to which these findings can generalize to interactions between a person and an IVA. For example, physical attraction between people could account for our finding that pairs with opposite genders have higher bonding, and these factors would presumably not be present in Human-VA interactions. However, to the extent that these findings generalize, they suggest that it may be most important to design an IVA to appear enthusiastic and attentive, rather than focusing on designing it to be agreeable, funny, attractive, or to have a similar age to the user. The importance of attentiveness may suggest that designing agents around mirroring (e.g. [3]) and contingent nonverbal cues (e.g. [4]) may be the most promising approaches.

\section{References}

1. H. van Vugt, J. Hoorn, and E. Konijn, "Interactive engagement with embodied agents: An empirically validated framework," Comp. Animation and Virtual Worlds, vol. 20, no. 2-3, pp. 195-204, 2009.

2. J. Wong and K. McGee, "Frown more, talk more: effects of facial expressions in establishing conversational rapport with virtual agents," in $I V A, 2012$, pp. 419-425.

3. S. Kahl and S. Kopp, "Modeling a social brain for interactive agents: integrating mirroring and mentalizing," in IVA, 2015, pp. 77-86.

4. J. Gratch et al., "Creating rapport with virtual agents," in IVA, 2007, pp. 125-138.

5. Zhao, Papangelis, and Cassell, "Towards a dyadic computational model of rapport management for human-virtual agent interaction," in $I V A, 2014$, pp. 514-527.

6. E. Butler, "Temporal interpersonal emotion systems the ties that form relationships," Pers. and Soc. Psych. Review, vol. 15, no. 4, pp. 367-393, 2011.

7. Cuperman and Ickes, "Big five predictors of behavior and perceptions in initial dyadic interactions," J. of Pers. and Soc. Psych., vol. 97, no. 4, pp. 667, 2009.

8. Charles S Carver and Michael F Scheier, Perspectives on personality, Pearson Higher Ed, 2011.

9. A. Horvath and L. Greenberg, "Development and validation of the working alliance inventory.," Journal of counseling psychology, vol. 36, no. 2, pp. 223, 1989.

10. M Astrid et al., "How our personality shapes our interactions with virtual characters-implications for research and development," in IVA, 2010, pp. 208-221.

11. Lewis R Goldberg, "The development of markers for the big-five factor structure.," Psychological assessment, vol. 4, no. 1, pp. 26, 1992. 